\title{
Dolor nociplástico: el nuevo descriptor
}

\section{Nociplastic pain: the new descriptor}

\author{
Tomás Nakazato-Nakamine ${ }^{1,2 *}$ y Pablo Quezada ${ }^{1,3}$ \\ ${ }^{1}$ Departamento Médico, Clínica Especializada en Dolor Muscular y del Hueso; ${ }^{2}$ Servicio de Medicina Física y Rehabilitación, Clínica Anglo \\ Americana; ${ }^{3}$ Servicio de Medicina Física y Rehabilitación, Hospital Aurelio Díaz Ufano. Lima, Perú
}

\section{Resumen}

En el año 2011, la International Association for the Study of Pain redefinió los dos descriptores utilizados hasta ese momento: el dolor nociceptivo (producido por la estimulación de nociceptores debido a un daño presente o potencial de tejidos no neurales), y el dolor neuropático (debido a una lesión o enfermedad del sistema nervioso somatosensorial). Al suprimir el término «disfunción» de la definición de este último, quedó en la nebulosa un gran grupo de pacientes que presentaba dolor por una alteración de la nocicepción (clínicamente evidente), pero que, al no poder demostrarse una activación de los nociceptores ni una lesión o enfermedad del sistema nervioso, no tenían cabida en la nueva dicotomía establecida (nociceptivo/neuropático). Se ha estimado que alrededor de dos tercios de los trastornos dolorosos musculoesqueléticos se encontraban fuera de ambas categorías (por ejemplo, los casos de lumbalgia y cervicalgia inespecíficas, fibromialgia, síndrome de dolor regional complejo tipo l, entre otros). Por ese motivo, en el 2017 se estableció un tercer descriptor: el dolor nociplástico. Con ello se ha podido subsanar el vacío existente, y dar finalmente una descripción fisiopatológica al diagnóstico de dolor crónico primario (sin daño estructural) que abarca a estos trastornos y que se encuentra codificado en la nueva Clasificación Internacional de Enfermedades, 11. ${ }^{a}$ revisión, (CIE-11).

Palabras clave: Dolor. Descriptor. Nociplástico. Nociceptivo. Neuropático.

\section{Abstract}

In the year 2011, the IASP (International Association for the Study of Pain) redefined the two descriptors: Nociceptive Pain (pain that arises from actual or threatened damage to non-neural tissue due to the activation of nociceptors), and Neuropathic Pain (pain caused by a lesion or disease of the somatosensory nervous system). By removing the term "dysfunction"from the definition of the latter, a huge group of patients that complained of pain as a result of an altered nociception (clinically evident) without a demonstrable activation of nociceptors nor a lesion or disease of the nervous system suddenly found them in a twilight zone, having no place in the new established dichotomy (nociceptive/neuropathic). It was estimated that about two thirds of the patients with musculoskeletal disorders could not be classified in any of these two descriptors (for example, non-specific low back and neck pain, fibromyalgia, complex regional pain syndrome type l, among others). For that reason, in 2017 a third descriptor was established: Nociplastic Pain. This new term has filled the existing gap, providing a physiopathological description for the diagnosis of Primary Chronic Pain (pain without structural damage), a term that has been codified in the new International Classification of Diseases (ICD-11) that encompasses all these cases.

Key words: Pain. Descriptor. Nociplastic. Nociceptive. Neuropathic.

\section{Correspondencia:}

*Tomás Nakazato-Nakamine

E-mail: tomasnakazato@yahoo.com
Disponible en internet: 19-06-2020 Rev Hosp Jua Mex. 2020;87(2):88-93

www.revistahospitaljuarez.com 1405-9622/@ 2020 Sociedad Médico-Quirúrgica del Hospital Juárez de México, A.C. Publicado por Permanyer. Este es un artículo open access bajo la licencia CC BY-NC-ND (http://creativecommons.org/licenses/by-nc-nd/4.0/). 


\section{Introducción}

El dolor musculoesquelético es la principal causa de consulta médica ${ }^{1}$ y la mayor fuente de discapacidad en el mundo². A pesar de ello, debido a que no suele tener relación con la mortalidad de la población, no llega a tener la repercusión mediática de otras condiciones tales como las enfermedades oncológicas, las infecciones o los trastornos cardiovasculares.

«Ud. no tiene nada, debe ser solo el estrés...» es un comentario común entre los médicos que tratan pacientes con dolor severo y que, al no encontrar ningún resultado positivo en los exámenes auxiliares, los catalogan como "psicosomáticos» 0 «simuladores», lo cual solo agrava su sufrimiento. ¿Aqué se debe esto? Lo que sucede es que la gran mayoría presenta dolor de origen «inespecífico» 0 «idiopático», los cuales son eufemismos médicos para decir que no tenemos idea acerca del agente causal del dolor que aqueja a estos pacientes. La lumbalgia, por ejemplo, es la causa más común de discapacidad en EE.UU. ${ }^{3}$, y se estima que alrededor del $70 \%$ de los pacientes que la presenta son de este tipo (inespecífico o idiopático $)^{4}$. En otras palabras: jsolo podemos identificar la etiología en menos de un tercio de los casos! (Fig. 1).

Lamayoría de los pacientes con trastornos inespecíficos suele frustrarse porque son sometidos a innumerables pruebas (muchas de ellas costosas) en busca de una lesión «objetiva» (radiografías, tomografías, resonancias magnéticas, ecografías, análisis de sangre, electromiografías, etc.), y son atiborrados de analgésicos cada vez más potentes, por periodos prolongados, y que les producen efectos adversos. A otros se les practican procedimientos invasivos o cirugías sin una clara indicación médica ante la desesperación por aliviar el dolor que experimentan o por la interpretación errónea de los exámenes auxiliares. Estos procedimientos terminan siendo inútiles y hasta contraproducentes debido a que no hay un daño o lesión estructural que pueda tratarse o repararse con dichas intervenciones. En la consulta de médicos especialistas en el tratamiento del dolor es muy común encontrar pacientes de este tipo.

\section{Los dos descriptores del dolor}

La International Association for the Study of Pain (IASP) es la sociedad internacional sin fines de lucro más importante en el mundo que estudia el fenómeno del dolor y que actualmente asesora a la Organización Mundial de la Salud en muchos aspectos en este campo. La definición vigente del dolor de esta entidad es: «Una

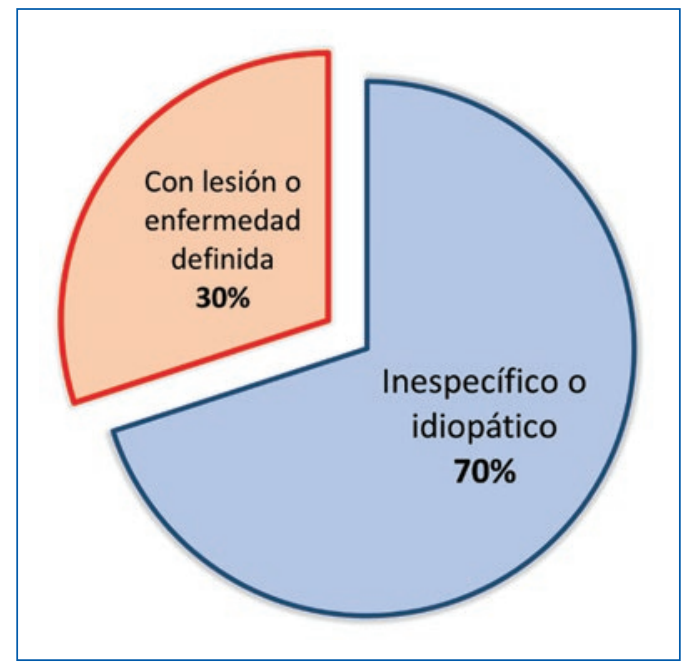

Figura 1. Dolor lumbar (fuente: Deyo RA, et al. ${ }^{4}$ ).

experiencia sensorial y emocional desagradable asociada a un daño tisular presente o potencial, o lo descrito en términos de esta» ${ }^{5}$. En el año 2005, ellos establecieron dos descriptores: 1) dolor nociceptivo, definido como el «producido por la estimulación de las terminaciones nerviosas nociceptivas primarias»; y 2) dolor neuropático, que era el «resultado de una lesión o disfunción del sistema nervioso». Este último es una actualización de la definición establecida previamente, en $1994^{6}$.

Ambos conceptos fueron revisados en 2011, y el dolor nociceptivo se redefinió como aquel «dolor que se produce por un daño real o potencial de tejidos no neurales y es debido a la activación de los nociceptores" (por ejemplo, una contusión o un desgarro muscular, osteoartritis o artrosis, inflamación debida a una enfermedad autoinmune como la artritis reumatoidea o el lupus eritematoso sistémico, neoplasias malignas que activan los nociceptores, etc.), y el dolor neuropático como el "dolor causado por una lesión o enfermedad del sistema nervioso somatosensorial» (por ejemplo, neuralgia postherpética, polineuropatía diabética, radiculopatía por hernia del núcleo pulposo, dolor por infarto talámico, etc.). La nota que acompañaba la definición del primero señalaba que este descriptor fue diseñado para diferenciarlo del segundo, es decir, que se debería utilizar para describir el dolor que se presentaba en un sistema nervioso somatosensorial con funcionamiento normal, en contraposición con los casos en lo que había una alteración de la función del sistema nervioso debida a una lesión o una enfermedad $^{7}$. Lo más destacado, sin embargo, fue que en esta última redefinición de dolor neuropático se suprimió el 
término «disfunción» del sistema nervioso que estaba en las descripciones de los años 1994 y 2005, por recomendación del Grupo de Interés Especial en Dolor Neuropático de la IASP8-10.

La supresión de este término dio como resultado que se excluyan muchas condiciones con alteraciones funcionales del sistema nervioso sin una lesión o enfermedad de este, creando una gran brecha entre la dicotomía dolor nociceptivo/dolor neuropático. Se dejaba, de este modo, sin un vocablo que describa los trastornos dolorosos sin activación de nociceptores ni lesión o enfermedad del sistema nervioso definida 0 probable, tales como la lumbalgia y la cervicalgia inespecífica o idiopática, el síndrome de fibromialgia, el síndrome del intestino irritable, el dolor regional crónico complejo tipo I, el síndrome de dolor miofascial y sensibilización espinal segmentaria, entre otros.

\section{El tercer descriptor}

Dada esta situación con dos descriptores (dolor nociceptivo y dolor neuropático) y una gran área gris en medio, se propuso un tercer término en el año $2016^{6}$. Los adjetivos que se plantearon fueron: «nociplástico», que reflejaba los cambios en la función de las vías nociceptivas; "algopático», que significaba una percepción o sensación alterada y patológica del dolor sin una lesión que lo produzca; y «nocipático», que denotaba un estado patológico o anormal de la nocicepción.

El nombre escogido por el Consejo de la IASP en 2017 fue el de «dolor nociplástico», el cual fue incluido en la terminología de esta entidad a fines de ese mismo año. Este se definió como el «dolor que se presenta como consecuencia de una alteración de la nocicepción a pesar de no haber una clara evidencia de un daño tisular presente o potencial que produzca la activación de nociceptores periféricos, ni evidencia de una lesión o enfermedad del sistema nervioso somatosensorial que origina el dolor» ${ }^{5,6}$.

Esta elección fue respaldada por abundante literatura que confirmaba la presencia de cambios en la actividad cerebral en las enfermedades anteriormente conocidas como "disfuncionales»"11,12. De este modo, a partir de ese momento se podía describir el dolor de los pacientes en que no había activación de nociceptores ni se había podido identificar una lesión o enfermedad del sistema nervioso somatosensorial (y que por lo tanto no calificaban para la definición de dolor nociceptivo ni neuropático).

Si tenemos en cuenta que alrededor de un tercio de la población adulta presenta dolor musculoesquelético, y que la gran mayoría de estos casos no tiene ningún hallazgo estructural de este sistema ${ }^{13}$, significa que muchos de ellos deberían estar catalogados dentro de este tercer descriptor. Por ello consideramos de gran importancia su inclusión en la terminología actual de la IASP.

\section{Discusión}

Muchos están en desacuerdo con el nuevo descriptor «dolor nociplástico», ya que su definición contiene términos vagos e imprecisos ${ }^{14}$. Se ha argumentado, además, que no ayuda a determinar el origen del dolor que experimentan los pacientes. Si bien se han podido describir cambios a nivel del sistema nervioso central en estos estados de alteración de la nocicepción, estos no pueden revelar a ciencia cierta por qué se presentan estos cuadros dolorosos.

Sin embargo, para nosotros, estos argumentos no desmerecen la utilidad del nuevo descriptor. Además, su definición no se presta a confusiones, ya que los pacientes que presentan dolor que puede describirse como nociceptivo oneuropático son excluidos de la misma. Ello evita también la superposición de términos, como ocurre con otro descriptor utilizado en la literatura médica, «dolor mixto» ${ }^{15}$, el cual es un término aún más discutible y que debería referirse solo a aquellos casos en donde efectivamente haya una combinación de dolor nociceptivo, dolor neuropático y/o dolor nociplástico en un mismo paciente. Además, este último no se encuentra en la terminología actual de la IASP, y somos de la opinión que es mejor evitarlo, y en lugar de este, los pacientes que verdaderamente presentan una combinación de los diferentes mecanismos los deberíamos describir como con dolor nociceptivo/neuropático o dolor nociceptivo/nociplástico, por ejemplo.

El término «nociplástico» se refiere a aquellos casos en los que se puede encontrar una alteración de la nocicepción; por lo tanto, este diagnóstico solo se aplica a los pacientes que refieren dolor con hipersensibilidad ${ }^{16}$ demostrada al menos clínicamente con hallazgos, tales como disestesias, hiperalgesia o alodinia, al examinar al paciente. Parece ser que la sensibilización del sistema nervioso central es el causante, ya que suele haber una respuesta favorable a los medicamentos que actúan a este nivel, tales como los anticonvulsivantes o los antidepresivos tricíclicos ${ }^{17}$. Estas características (la presencia de signos clínicos y la respuesta a los medicamentos de acción central) son lo que diferencia claramente a los pacientes con nocicepción alterada (dolor nociplástico) de aquellos en los que 
Tabla 1. Clasificación del dolor

\begin{tabular}{|c|c|c|c|}
\hline Descriptor* & Definición & Notas & Ejemplos \\
\hline \multicolumn{4}{|c|}{ Incorporados en la taxonomía de la IASP (hasta el 2020): } \\
\hline Dolor nociceptivo & $\begin{array}{l}\text { Dolor que se presenta por un daño } \\
\text { real o potencial de los tejidos no } \\
\text { neurales y se debe a la activación } \\
\text { de nociceptores. }\end{array}$ & $\begin{array}{l}\text { El sistema nervioso } \\
\text { somatosensorial debe tener } \\
\text { funcionamiento normal (intacto). }\end{array}$ & $\begin{array}{l}\text { Dolor por artrosis (osteoartritis), } \\
\text { enfermedades autoinmunes (artritis } \\
\text { reumatoide, lupus eritematoso, etc.), } \\
\text { dolor oncológico que activa } \\
\text { nociceptores. }\end{array}$ \\
\hline $\begin{array}{l}\text { Dolor } \\
\text { neuropático }\end{array}$ & $\begin{array}{l}\text { Dolor ocasionado por una lesión o } \\
\text { enfermedad del sistema nervioso } \\
\text { somatosensorial. }\end{array}$ & $\begin{array}{l}\text { Requiere una lesión o enfermedad } \\
\text { demostrable del sistema nervioso } \\
\text { somatosensorial que satisfaga } \\
\text { criterios diagnósticos neurológicos } \\
\text { establecidos. }\end{array}$ & $\begin{array}{l}\text { Neuralgia postherpética, } \\
\text { polineuropatía diabética, } \\
\text { radiculopatía, dolor regional crónico } \\
\text { complejo tipo II. }\end{array}$ \\
\hline $\begin{array}{l}\text { Dolor } \\
\text { nociplástico }\end{array}$ & $\begin{array}{l}\text { Dolor que se presenta por una } \\
\text { nocicepción alterada a pesar de no } \\
\text { haber una clara evidencia de un } \\
\text { daño real o potencial tisular que } \\
\text { ocasione la activación de los } \\
\text { nociceptores periféricos, o evidencia } \\
\text { de una lesión o enfermedad del } \\
\text { sistema nervioso somatosensorial } \\
\text { que ocasione el dolor. }\end{array}$ & $\begin{array}{l}\text { Describe el dolor con hallazgos } \\
\text { clínicos que sugieren una } \\
\text { alteración de la nocicepción, a } \\
\text { pesar de que no haya una clara } \\
\text { evidencia de enfermedad o lesión } \\
\text { del sistema somatosensorial. No } \\
\text { es un sinónimo de "sensibilización } \\
\text { central», el cual es un concepto } \\
\text { neurofisiológico, ni tampoco del } \\
\text { dolor "psicógeno". }\end{array}$ & $\begin{array}{l}\text { Síndrome de fibromialgia, dolor } \\
\text { regional crónico complejo tipo I, } \\
\text { lumbalgia inespecífica, cervicalgia } \\
\text { inespecífica, síndrome de dolor } \\
\text { miofascial, síndrome de } \\
\text { sensibilización espinal segmentaria. }\end{array}$ \\
\hline
\end{tabular}

\section{Aún no incorporados en la taxonomía de la IASP:}

\begin{tabular}{|c|c|c|c|}
\hline Dolor mixto & $\begin{array}{l}\text { Dolor por superposición de los } \\
\text { diferentes tipos de dolor } \\
\text { (nociceptivo, neuropático, } \\
\text { nociplástico) en cualquier } \\
\text { combinación. }\end{array}$ & $\begin{array}{l}\text { Pueden estar actuando en forma } \\
\text { simultánea y/o concurrente en una } \\
\text { región determinada del cuerpo. } \\
\text { Cada uno de ellos puede ser el } \\
\text { preponderante en un punto } \\
\text { determinado de tiempo. }\end{array}$ & $\begin{array}{l}\text { Pacientes oncológicos con tur } \\
\text { que dañan estructuras somáti } \\
\text { del sistema nervioso; paciente } \\
\text { artritis reumatoidea y que ade } \\
\text { presentan síndrome de fibrom }\end{array}$ \\
\hline $\begin{array}{l}\text { Dolor de origen } \\
\text { desconocido }\end{array}$ & $\begin{array}{l}\text { Dolor de causa y origen } \\
\text { desconocidos. }\end{array}$ & $\begin{array}{l}\text { Dolor que no puede clasificarse } \\
\text { como nociceptivo, neuropático o } \\
\text { nociplástico. }\end{array}$ & $\begin{array}{l}\text { Pacientes con síndrome } \\
\text { somatomorfo, depresión asoci } \\
\text { dolor, dolor sin evidencias clín } \\
\text { ni de exámenes auxiliares. }\end{array}$ \\
\hline
\end{tabular}

los mecanismos biomédicos son desconocidos, que se les debe catalogar más bien con la descripción de «dolor de origen desconocido». Este tipo de dolor tampoco está incluido aún en la terminología de la IASP, pero consideramos que es necesario utilizarlo para aquellos casos en que no se puede catalogar al paciente en ninguno de los tres descriptores establecidos ${ }^{6}$. Estos serían los verdaderos casos de dolor idiopático. La tabla 1 detalla los descriptores de dolor, según Kosek, et al. ${ }^{6}$ y Freynhagen, et al. ${ }^{15}$. La figura 2 representa el dolor mixto como la superposición de los tres descriptores definidos por la IASP, y el dolor de origen desconocido sería una categoría aún por definir.
¿Por qué este tercer descriptor es tan controversial? Los pacientes que no tienen una lesión objetivable (no cumplen con los criterios de dolor nociceptivo ni neuropático), y que suele ser la mayoría de los casos de dolor musculoesquelético crónico, no son manejados adecuadamente en la práctica clínica actual. Estos pacientes suelen ser derivados por los médicos generales a especialidades quirúrgicas para su tratamiento, tales como la ortopedia y traumatología, o la neurocirugía. Pero si no hay una estructura dañada, no hay nada que operar, y por lo tanto es poco lo que estos pueden hacer. Otros son derivados a reumatología, pero al no haber un proceso inflamatorio o autoinmune en estos casos, tampoco 


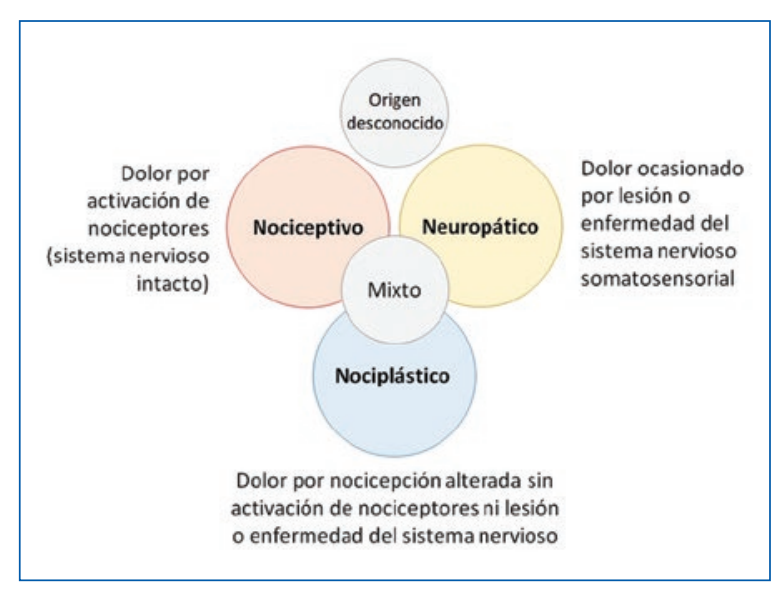

Figura 2. Tipos de dolor (adaptado de Freynhagen $R$, et $a{ }^{19}{ }^{19}$ ).

es mucho lo que esta especialidad puede resolver. Para agravar el problema, ahora se han puesto de moda sofisticados estudios de imagen, tales como la tomografía axial multicorte con reconstrucción en tercera dimensión, la resonancia magnética nuclear con contraste o la ecografía musculoesquelética de alta resolución, los cuales pueden dar imágenes estructurales impresionantes, pero que, como se intuye ahora, siguen siendo inútiles para el diagnóstico de dolor nociplástico. Todo ello explica el por qué hay una incomprensión y hasta un rechazo por el nuevo descriptor en la comunidad médica, y muchos piensan aún hasta ahora que «no puede ser que el paciente se queje tanto del dolor y no se le encuentre nada (en los exámenes auxiliares)".

Por este motivo, se entiende también que haya una proliferación de tratamientos «alternativos» 0 «complementarios", tales como la quiropraxia, osteopatía, acupuntura tradicional, masoterapia, homeopatía, etc., que tratan de llenar dicho vacío a pesar de su falta de adecuación de la fisiopatología y la medicina convencional. Muchos de estos tratamientos aún están en el proceso de validación de acuerdo con el método científico, pero son aceptados por innumerables pacientes al brindarles opciones terapéuticas muy discutibles desde el punto de vista médico, pero mucho más empáticas, y que les evitan la marginación soterrada a la que son expuestos cuando acuden a los sistemas de salud formales. Pese a ello, nosotros no estamos a favor de estos tratamientos sin una adecuada supervisión médica, debido a su falta de rigor científico y a la posibilidad de poner en riesgo la salud de los pacientes, ya que estos pueden necesitar evaluaciones médicas para descartar trastornos más serios (como neoplasias, infecciones, fracturas ocultas, enfermedades autoinmunes, entre otros). La medicina física y rehabilitación es una especialidad médica con fundamento científico y que forma parte del sistema de salud formal (a nivel hospitalario), y es donde consideramos que deberían remitirse estos casos de dolor musculoesquelético crónico nociplástico. La presencia de este nuevo descriptor será muy útil para poder catalogar a los pacientes sin el estigma que representan los términos «inespecífico» o «idiopático" en la actualidad, y con ello se evitará que sean marginados por la medicina convencional.

¿Es el dolor nociplástico un tipo de dolor psicógeno? Es bien sabida desde hace mucho tiempo la existencia de una pobre correlación entre la patología periférica y los síntomas dolorosos de los pacientes, y está más clara, en cambio, la existencia de una modulación alterada en el sistema nervioso en los casos de dolor crónico sin daño estructural. El uso del descriptor dolor nociplástico es, por estos motivos, muy útil a pesar de sus controversias, al evitar la tendencia de los médicos a pensar que el dolor del paciente no es real, sino más bien una forma de sensibilización del sistema nervioso como mecanismo fisiopatológico. El mayor avance que se espera con la difusión de este nuevo término es evitar que los médicos les sigan diciendo a sus pacientes que «todo está en su mente»18. En la actualidad, se debe dejar de lado el concepto de que existe un dolor psicógeno en contraposición a uno somático, porque incluso en las enfermedades mentales, tales como la esquizofrenia o la depresión, los avances en el campo de la neurociencia nos hacen ver que estos cuadros ya no son considerados como trastornos puramente no somáticos. Todo dolor tiene, en realidad, un componente multifactorial («bio-psico-social») en donde las reacciones cerebrales neurofisiológicas como producto de las emociones y las interacciones con el ambiente externo contribuyen en gran medida a los cambios en la percepción de este. Por ello, debemos eliminar el estigma que representa clasificar a los pacientes con dolor crónico sin lesiones objetivas como enfermos mentales, ya que los procesos psicológicos pueden influir, pero no suelen determinar, la causa del dolor en la gran mayoría de los casos. Por este motivo, la nueva Clasificación Internacional de Enfermedades, $11 .^{\text {a }}$ revisión, (CIE-11) ha codificado a todos los dolores crónicos, incluyendo el dolor crónico primario, fuera de la esfera de los trastornos psiquiátricos ${ }^{19-21}$.

\section{Conclusión}

El término «dolor nociplástico» es un nuevo descriptor que servirá para promover un monitoreo sistemático 
de la función nociceptiva alterada, especialmente en pacientes que presentan dolor crónico que no encaja en las definiciones de dolor nociceptivo ni neuropático. Ello evitará que aquellos que presenten trastornos dolorosos inespecíficos (con un cuadro clínico objetivo, pero sin una lesión o enfermedad demostrable en exámenes auxiliares) queden relegados de la atención médica formal, a la vez que permitirá realizar trabajos de investigación sobre este tópico en particular. También será muy útil para describir a los pacientes con trastornos dolorosos que se engloban dentro del diagnóstico «dolor crónico primario» (dolor sin daño estructural) que está codificado en el nuevo CIE-11.

\section{Conflicto de intereses}

El autor principal ha sido nombrado speaker de Laboratorios Grünenthal a partir del mes de febrero del 2020 pero no ha recibido, hasta el momento de presentar el artículo, ninguna participación económica de dicha empresa.

El segundo autor declara no tener ningún conflicto de intereses.

\section{Financiamiento}

Los autores no recibieron patrocinio para llevar a cabo este artículo.

\section{Responsabilidades éticas}

Protección de personas y animales. Los autores declaran que para esta investigación no se han realizado experimentos en seres humanos ni en animales.

Confidencialidad de los datos. Los autores declaran que han seguido los protocolos de su centro de trabajo sobre la publicación de datos de pacientes.
Derecho a la privacidad y consentimiento informado. Los autores declaran que en este artículo no aparecen datos de pacientes.

\section{Bibliografía}

1. Deyo RA, Rainville J, Kent DL. What can the history and physical examination tell us about low back pain? JAMA. 1992; 268(6):760-5.

2. Rice AS, Smith BH, Blyth FM. Pain and the global burden of disease. PAIN. 2016; 157:791-6.

3. The US Burden of Disease Collaborators. The State of US Health, 19902016. JAMA. 2018; 319(14):1444-72.

4. Deyo RA, Weinstein JN. Low Back Pain. N Engl J Med. 2001;344:(5):36370.

5. IASP. iasp-pain.org. [Online]. (Citado el 22 de octubre de 2019). Disponible en: https://www.iasp-pain.org/Education/Content.aspx?ItemNumber $=1698$.

6. Kosek E, Cohen M, Baron R, Gebhart GF, Mico JA, Rice A, et al. Do we need a third mechanistic descriptor for chronic pain states? Pain. 2016;157:1382-6.

7. Trouvin AP, Perrot S. New concepts of pain. Best Pract Res Clin Rheumatol. 2019;33(3):101415.

8. Treede RD, Jensen TS, Campbell JN, Gruccu G, Dostrovsky JO, Griffin JW, et al. Neuropathic pain: Redefinition and a grading system for clinical and research purposes. Neurology. 2008;70(18):1630-5.

9. Jensen TS, Baron R, Haanpää M, Kalso E, Loeser JD, Rice AS, et al. A new definition of neuropathic pain. Pain. 2011;152:2204-5.

10. Haanpää ML, Treede RD. Diagnosis and Classification of Neuropathic Pain. Pain: Clinical Update. 2010;18(7).

11. Giesecke $T$, Gracely $R H$, Grant MA, Nachemson A, Petzke F, Williams DA, et al. Evidence of augmented central pain processing in idiopatic chronic low back pain. Arthritis Rheum. 2004;50:613-23.

12. Burgmer M, Pleiderer B, Maihöfner C, Gaubitz M, Wessolleck E, Heuft G, et al. Cerebral mechanismos of experimental hyperalgesia in fibromyalgia. Eur J Pain. 2012;16:636-47.

13. Bergman S. Management of musculoskeletal pain. Best Practice \& Research Clinical Rheumatology. 2007;21(1):153-66.

14. Granan LP. We do not need a third mechanistic descriptor for chronic pain states! Not yet. Pain. 2017;158:179.

15. Freynhagen R, Arevalo H, Calderón-Ospina C, Chen J, Rakhmawati ED, Fernández-Villacorta FJ, et al. Current understanding of the mixed pain concept: a brief narrative review. Current Medical Research and Opinion. 2019;35(6):1011-8.

16. Kosek E, Cohen M, Baron R, Mico JA, Rice A. Reply. Pain. 2017;158:180.

17. Woolf CJ. Central sensitization: Implications for the diagnosis and treatment of pain. Pain. 2011;152(3):S2-15.

18. Andrews N. Pain Research Forum. [Online]; 2018 (Citado el 4 de marzo de 2020). Disponible en: https://www.painresearchforum.org/news/92059whats-name-chronic-pain.

19. Harris NL. Part I: Proposed New Classifications And Nomenclatures For Chronic Pain. Reflectios. RGA's Global Medical Newsletter. 2019; 47:2-8.

20. Treede RD, Rief W, Barke A, Aziz Q, Bennett MI, Benoliel R, et al. Chronic pain as a symptom or a disease: the IASP Classification of Chronic Pain for the International Classification of Diseases (ICD-11). Pain. 2019;160(1):19-27.

21. Organización Mundial de la Salud (OMS). CIE-11. Clasificación Internacional de Enfermedades, 11.a revisión. [Online]. (Citado el 4 de marzo de 2020). Disponible en: https://icd.who.int/browse11/l-m/es\#/http://id. who.int/icd/entity/1581976053. 